\title{
Leaked Expressions Captured with Wearable High Resolution Facial Electromyography
}

\author{
Omer Granoviter $^{1 *}$ and Yael Hanein ${ }^{1}$ \\ ${ }^{1}$ School of Electrical Engineering. \\ *Corresponding author. E-mail: omer.gra@gmail.com
}

(Received xx xxx xxxx)

Keywords: Leaked expressions; facial expressions; skin electrode; electromyography.

\begin{abstract}
Facial-expressions play a major role in human communication and provide a window to an individual's emotional state. While facial expressions can be consciously manipulated to conceal true emotions, very brief leaked expressions may occur, exposing one's true internal state. Leaked expressions are therefore considered as an important hallmark in deception detection, a field with enormous social and economic impact. Challengingly, capturing these subtle and brief expressions has been so far limited to visual examination (manual or machine-based), with almost no electromyography evidence. In this investigation we set to explore whether electromyography of leaked expressions can be faithfully recorded with specially designed wearable electrodes. Indeed, using soft multi-electrode array based facial electromyography, we were able to record localized and brief signals in individuals instructed to suppress smiles. The electromyography evidence was validated with high-speed video recordings. The recording approach reported here provides a new and sensitive tool for leaked expression investigations and a basis for improved automated systems.
\end{abstract}

\section{Introduction}

Facial expressions are an important part of human non-verbal communication. Studied extensively over decades (10), facial expressions became an important element in many fields, including child development, psychological evaluation and neurological disorders (11). Facial expressions are considered universal, and yet, they are extremely complicated, with a single facial expression involving multiple muscles. Challengingly, facial expressions can be consciously manipulated. According to Ekman and Friesen, there are three major ways in which manipulation can take place: The face may be simulated, masked or neutralized. An expression is considered simulated if there is no underlying emotion accompanying it, masked if an emotion is concealed by using a facial expression with no correspondence to underlying emotion, and neutralized when a facial expression is inhibited while the face remains neutral (17), (12).

Despite a conscious effort to manipulate a facial expression, leaked expressions may take place. Leaked expressions are especially intriguing and challenging to investigate. In particular, microexpressions (MEs), which were first detected by Haggard and Isaacs in 1966 (18), proved elusive. Ekman and Friesen formally defined them as particularly brief facial expressions that occur when an emotion is being concealed (16), (13). Although controversy exists regarding the length of MEs, it is mainly generally accepted to regard the upper limit as $0.5 \mathrm{~s}(37)$. MEs may be elicited either subconsciously or if a subject has become aware of an expression and tries to repress it (19).

Efforts to detect brief leaked expressions and in particular ME are almost entirely based on computer vision (CV) detection. Several spontaneous ME databases have emerged in recent years (37), (35),(27),

C Cambridge University Press 2019. This is an Open Access article, distributed under the terms of the Creative Commons Attribution licence (http:// creativecommons.org/licenses/by/4.0/), which permits unrestricted re-use, distribution, and reproduction in any medium, provided the original work is properly cited. 
Table 1. Video's duration, size and description

\begin{tabular}{|ccc|}
\hline Duration [s] & Size width and height [pixels] & Description \\
\hline \hline 9.81 & {$[1920,1080]$} & Funny animals \\
23.4 & {$[1920,1080]$} & Parents ate children's candy \\
29.18 & {$[1920,1080]$} & Parents ate children's candy \\
16.73 & {$[1920,1080]$} & Parents ate children's candy \\
332.2 & {$[1920,1080]$} & Piper \\
58.56 & {$[1920,1080]$} & What a woman wants - Pantyhose scene \\
81.88 & {$[1920,1080]$} & When Harry Met Sally - Fake Orgasm Scene \\
29.92 & {$[568,320]$} & Boy loses running race \\
410.69 & {$[1920,1080]$} & Dustin \\
390.89 & {$[1920,1080]$} & Feast \\
11.29 & {$[1920,1080]$} & Women trying to catch a cat \\
10.99 & {$[1920,1080]$} & Cat in the bathtub \\
9.37 & {$[1920,1080]$} & Cat scares girl \\
5.61 & {$[1920,1080]$} & Cat scared of water \\
28.67 & {$[1920,1080]$} & Parents ate children's candy \\
18.2 & {$[1920,1080]$} & Parents ate children's candy \\
& & \\
\hline
\end{tabular}

providing important input for new algorithms. The main disadvantages of the CV methods are high computational time (frame by frame analysis with high frame rate videos), multiple parameters needed for calibration, the need to track subject movements, the need for a frontal view, high frame rate and a well-lit environment. In a review article, Merghani et al.(30) described a total of 47 algorithms from the past decade, noting that differences in frame rates, coding styles and the number of participants vary considerably between studies.

Facial surface electromyography (sEMG) investigations, in the context of leaked and ME are scarce (31), further contributing to the elusiveness of these expressions. It is clear that an improved tool suitable to detect leaked and ME is vital for better understanding and utilization of this intriguing phenomena. To address this challenge, in the investigation reported below we applied newly developed soft multi electrode arrays to capture localized and short leaked expressions. Importantly, the electrodes allow participants to perform natural head and facial movements. We describe sEMG recordings and discuss how our results compare with CV based recordings and analysis of leaked expressions.

\section{Methods}

\subsection{Participants}

Nine subjects ( 8 females, average age $25.2 \pm 6.5$ ) were recruited and their facial EMG was recorded. All subjects were given 75 NIS for participating in the entire experiment, which lasted an hour and a half. Subjects signed relevant consent forms in accordance to the Tel Aviv University Ethics Committee.

\subsection{Task}

The Experiment lasted a total of an hour and a half and was devised into two parts. The first, a calibration designed to measure SNR and to ensure that all electrodes were recording correctly. The second, an experiment designed to elicit leaked micro-expressions. Before initiating the calibration, each subject was shown four photographs and text segments depicting four expressions: smile, close-the eyes-forcefully, contract-the-eyebrows and press-the-lips-together (Figure 2). The experimenter then validated that the subjects were doing each facial expression correctly prior to initiating the calibration. 
(a)

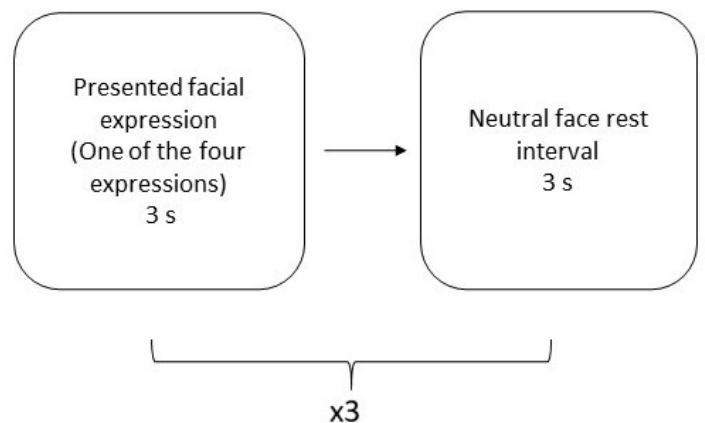

(b)

Experiment phase

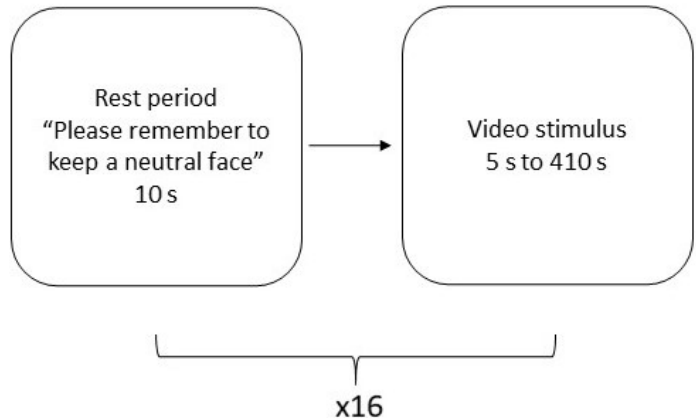

Figure 1. Experiment Task. (a) Calibration phase. Each subject was requested to smile, close-the-eyes-forcefully, contract-the-eyebrows and press-the-lips-together for $3 \mathrm{~s}$, followed by a rest interval of a neutral expression lasting $3 \mathrm{~s}$. Each facial expression was repeated 3 times consecutively, amounting to a total of 12 facial expressions in total. (b) Experiment phase. Each subject was reminded of the experiment's instruction "Please remember to keep a neutral face" for $10 \mathrm{~s}$, followed by a video designed to elicit smiles. Duration of the videos were between 5 and $410 \mathrm{~s}$.

Each expression was then presented for $3 \mathrm{~s}$ following a $3 \mathrm{~s}$ neutral expression interval and repeated three times to a total of 12 facial expressions. The second phase, consisted of a task adopted from previous studies designed to elicit leaked micro-expressions for the purpose of database creation or detection $(26 ; 31 ; 27 ; 36 ; 6)$. The subjects were seated in front of a computer screen, and watched 16 videos, amounting to 24.45 minutes, of positive valence designed to elicit smiles (videos are described in Table 1). In order to create a situation where leaked expressions occur, each subject was given instructions to retain a neutral expression. The task consisted of displaying a video followed by a rest interval of 10 s. During the rest interval subjects were reminded of the experiment instructions by seeing the caption "Please remember to keep a neutral face" as depicted in Figure 1.

\subsection{Stimuli}

Videos were tested on two pilot subjects and elicited 13 visually confirmed leaked expressions. All videos were taken from YouTube. Movie lengths varied from 5 to 410 s, resolution varied from 568x320 to $1920 \times 1080$ pixels (see Table 1. All videos were presented at 30 frames per second.

\subsection{Recording setup}

Electrode arrays were fabricated using a procedure detailed before $(3 ; 23)$. Briefly, electrode arrays were printed on a thin $(80 \mu \mathrm{m})$ polyurethane (PU) substrate with a two-step process of silver traces $(50 \mu \mathrm{m})$ and carbon electrodes ( $20 \mu \mathrm{m}$ thick). A thin double adhesive passivation layer $(60 \mu \mathrm{m})$ was used (22). The electrode diameter was $5 \mathrm{~mm}$. The electrode arrays were connected to two amplifier units (Intan Technologies amplifier evaluation board, RHD2000) using a custom-made printed circuit board (PCB) and a zero-insertion-force (ZIF) connector. The amplifier was attached to the subject's cheek using a medical tape (3M, Transpore).The RHD2132 system was used in this study. It has 32 low-noise amplifiers (192 gain). The output of the amplifier is digitized with a 16 bit Analog-to-Digital converter (ADC). Electrode arrays placement was set according to FACS AU's mostly associated with positive emotions (with the exception of the Corrugator supercilii associated with frowning) (14). The first array provides recording at the tip of the lip addressing Orbicularis Oris and was placed at the right side of the subject's face. The second array was placed on the left side of the face at close proximity to the Zygomaticus Major (electrodes 2-4), Orbicularis Occuli (elecrode 4-5) and Corrugator supercilii (electrodes 6-7) muscles area $(23 ; 20)$. A commercial ground plate electrode (Natus) was positioned on the bony are by the seventh vertebra (C7). The sEMG data was recorded at $3000 \mathrm{~S} / \mathrm{s}$. The subjects' 

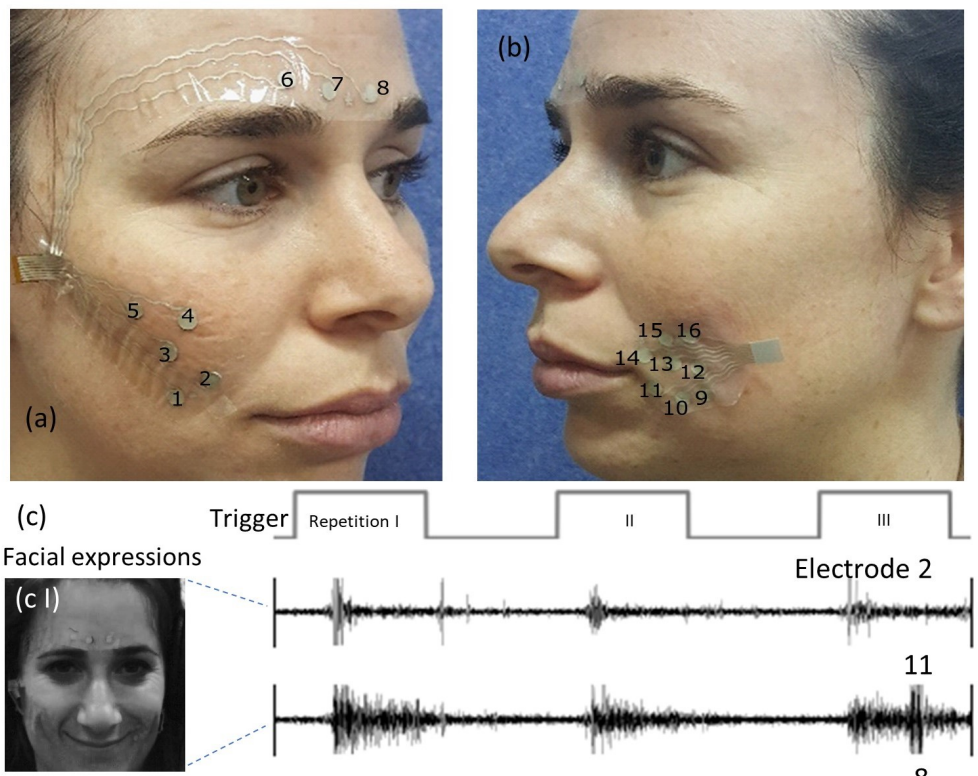

Electrode 2
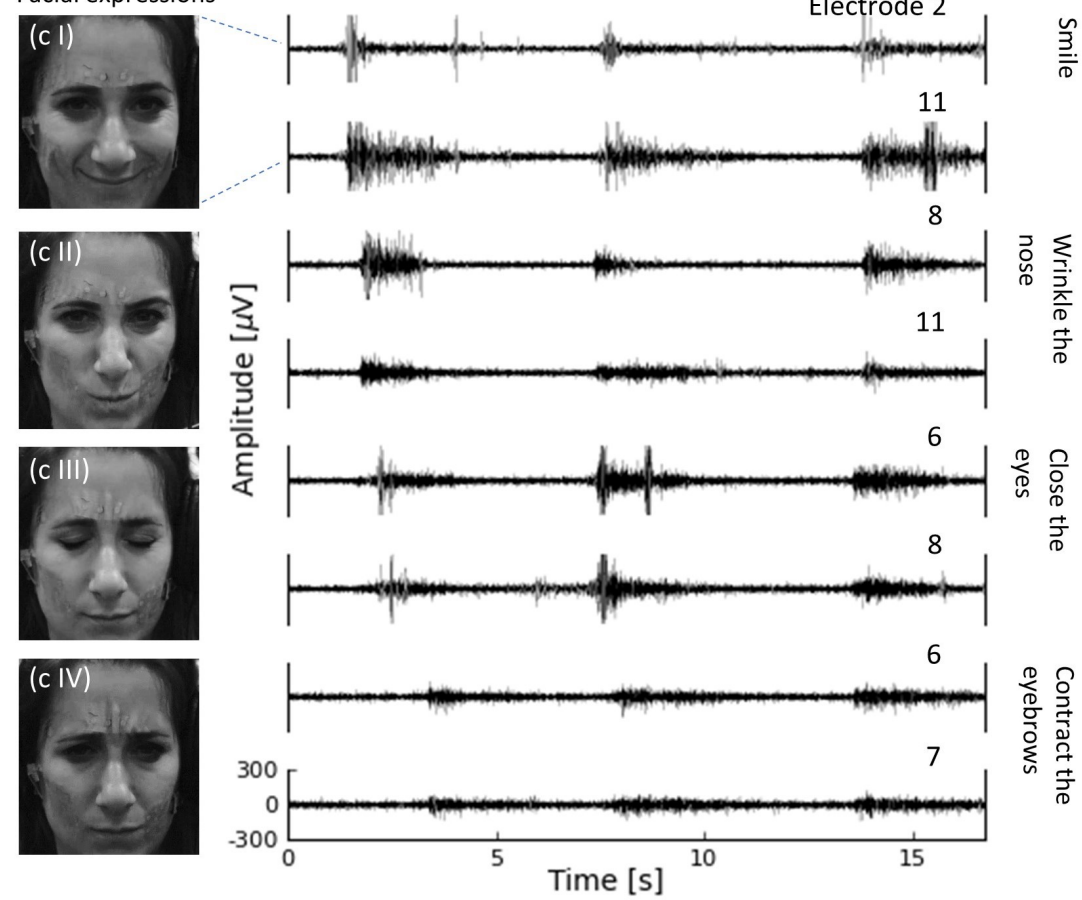

Figure 2. Soft electrode arrays positions and numbering on (a) right and (b) left sides of the face. (c) Calibration data of four voluntary facial expressions: (c I) smile, (c II) wrinkling of the nose, (c III) closing the eyes forcefully and (c IV) contracting the eyebrows. Command timing (trigger) is the top curve, each facial expression was repeated three times.

facial expressions were also recorded using a MAKO U-130B high-speed camera at 100 frames/s and 1280x 1024 resolution.

\subsection{Data analysis}

Out of the 9 recorded data sets, 4 were fully analyzed. Data from two subjects had noisy recordings, one subject had a corrupted data file, one subject had a corrupted recorded video and one subject was extremely tired, and fell asleep during multiple segments of the experiment. Data was first filtered using a comb filter for $50 \mathrm{~Hz}$ harmonics ( 50 to $250 \mathrm{~Hz}$ ) and a band-pass filter in the frequency range of 20-250 Hz. Filtering the signal below the frequency of $20 \mathrm{~Hz}$ was necessary to reduce the baseline noise 
and enable the detection of minute expressions. For the purpose of events detection, the authors implemented the modified adaptive linear energy detector (M-ALED)(4). For the purpose of confirming these leaked expressions, a graphical user interface was built with the ability to display both the recorded video and sEMG simultaneously, including an annotation panel to provide time stamped labels. Each found event was then reviewed and events of mechanical noise or other facial expressions un-related to the stimuli (e.g. yawning, sneezing) were removed, leaving only events containing a clear visually confirmed facial expression (smile). Time duration for each event was measured on the sEMG data using the graphical user interface labeling panel. Due to the manual nature of the duration's measurements, a measuring error should be considered. Spectral analysis (seen in Figure 3) was done using a sliding window of 1024 samples (approx. 0.3413 [s]), with 1000 samples overlap. Detection of lip contours for the purpose of visual presentation (seen in Figure 3) was done using the canny edge detector (8).

\section{Results}

Two electrode arrays were placed on the right and left sides of the face of each subject (Figure 1). Visual data and sEMG were collected while subjects were following text instructions and watching movies on a computer display. Initially, the participants were instructed to imitate four facial expressions, each repeated for three times: Closing the eyes forcefully, wrinkling the nose, smiling and contracting the eyebrows (see Figure 1). Each expression lasted $3 \mathrm{~s}$ and was followed by a rest period of $3 \mathrm{~s}$.

Data from the calibration step show high signal-to-noise ratios on single-ended data channels. Values as high as 12, 4.5, 20 and 5.5 were recorded for closing the eyes, contracting the eyebrows, smiling, and wrinkling the nose, respectively. Typical baseline noise was $20 \mu \mathrm{V}$ (after filtering) in almost all electrodes (Figure 2).

Subjects facial sEMG was then recorded during a session aimed to elicit leaked expressions. The participants were presented with positive funny videos (Table 1) and were requested and reminded throughout the session to maintain a neutral face. sEMG events were isolated and only events that were visually validated using the video remained for analysis (elaborated in the data-analysis section). The four subjects (that their data were analyzed) elicited a total of 48 leaked expressions, ranging in duration between 0.160 to $12.02 \mathrm{~s}$ and sEMG amplitudes in the range of 82 to $990 \mu \mathrm{V}$. We assume that the long and high amplitude expressions took place when the subjects forgot to follow the instructions or could not suppress laughter or smiles. This point will be further discussed below. For clarity, we refer to all recorded expressions in the second session as "leaked".

Figure 3 shows two examples of leaked expressions (long and short) along with a video analysis of the short leaked expression (lip contour). A short symmetric smile (below $0.5 \mathrm{~s}$ ) and a long smile (above $0.5 \mathrm{~s}$ ). A clear difference in amplitude and the number of activated electrodes is apparent. In Figure 3 (a) a long leaked expression $(>0.5 \mathrm{~s})$ is typified with activity in all electrodes located around the Orbicularis Oris, Risorius and Zygomaticus Major, while Figure 3 (b) shows a short leaked expression $(<0.5 \mathrm{~s})$, with activity mainly in electrodes $1-5$ and 13 . Figure 3 (e) shows the lip contour of the specific chosen short leaked expression with the corresponding lip contours of two frames. A single frame before the change in expression began (termed the onset frame) and the frame in which the facial expression is at full capacity (termed the apex frame).

One of the most important features of leaked expressions is their brief nature. In Figure 4 we show the histogram of signal duration and amplitude. A clear group of leaked expressions can be identified below $0.5 \mathrm{~s}$ and $300 \mu \mathrm{V}$. The visible group of smiles with durations below $0.5 \mathrm{~s}$, is with accordance to both Haggard and Isaacs (18) and later Ekman and Freisen (16) discovery of a group of leaked expressions termed micro-expressions.

Altogether, sEMG data shows large inter and intra subject variability. While there are evident common features between subjects, there are instances of increased activity in several electrodes and instances of an extremely localized signal. 

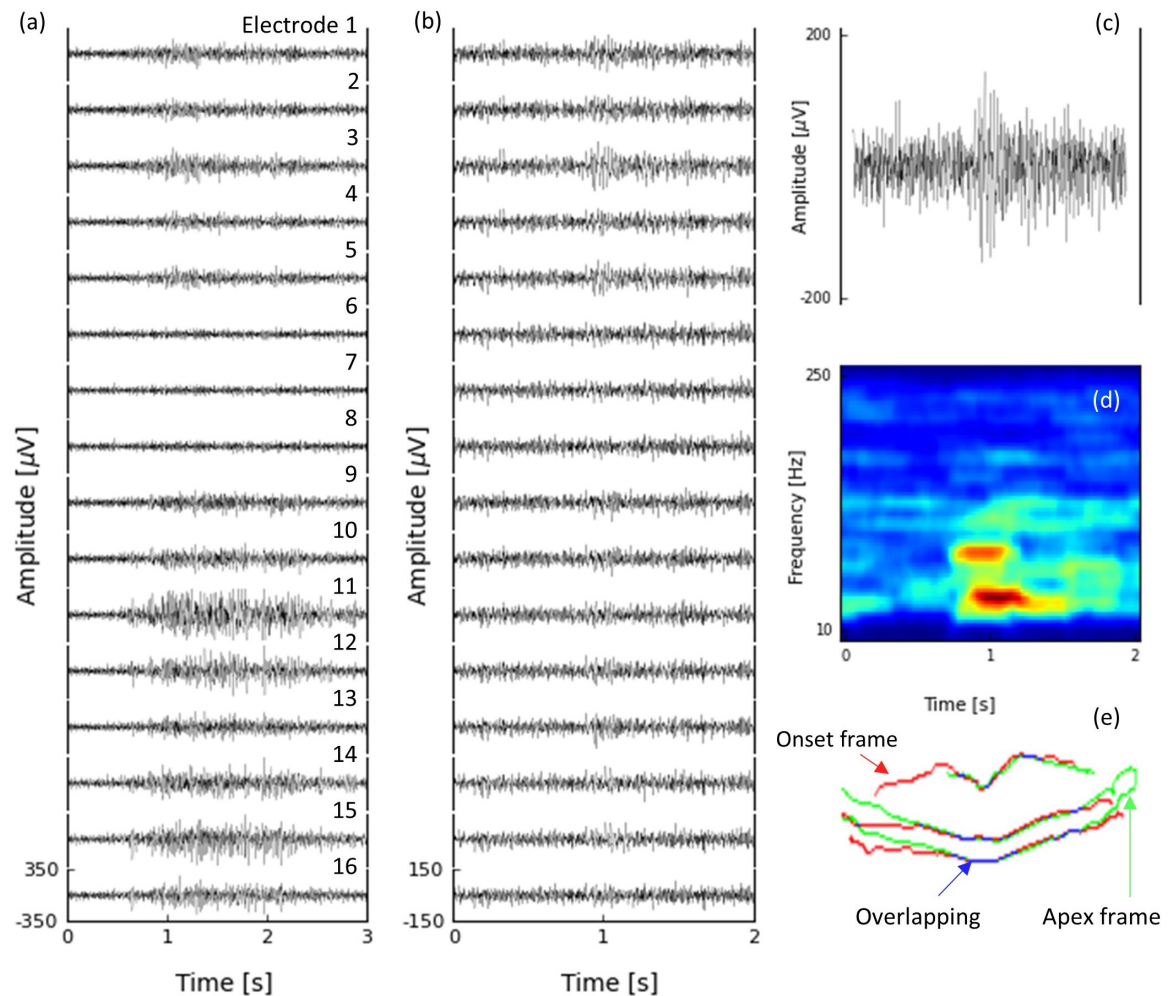

Figure 3. Leaked smiles. (a) A large leaked expression (above $0.5 \mathrm{~s}$ ) of a symmetrical smile with high amplitude and multiple electrode activations on both sides of the face. (b) A short leaked expression (below $0.5 \mathrm{~s}$ ) with lower amplitudes and electrode activations on both sides of the face. (c) The short leaked expression sEMG in the most prominent electrode (number 3). (d) A spectrogram of the most prominent electrode. (e) Lip contour segmentation on first onset frame (red), and first apex frame (green) and overlapping pixels (blue).

In order to visualize the variability, we analyzed the number of activated electrodes to show a considerable variability between the 4 analyzed subjects (see Figure 5). In particular, it is interesting to investigate the variability of the leaked expressions in the two visible groups seen in figure 4 . For leaked expressions longer than $0.5 \mathrm{~s}$, all subjects exhibited activation in almost all electrodes (1-6 and 9-16, with a single case including activation in electrodes 6-8). When considering activations below $0.5 \mathrm{~s}$, the variability is more apparent. Subject 1 exhibited activation on channel 14, with a single event exhibited on more electrodes (1-6, 9-16), a total of 5/6 events were on a single electrode (localized activation). Subject two exhibited localized activation in $3 / 3$ events. Subject 3 exhibited a total of 1/3 localized activations, while subject 4 exhibited a total of $2 / 13$ localized activations. It is interesting to note that the subject that exhibited the most leaked expressions below $0.5 \mathrm{~s}$ exhibited the most un-localized event ratio (two or more electrodes activated). It is noticeable that electrodes 6-8 were not activated at all except in one micro and one macro expressions. This is expected as these electrodes are in close proximity to the Corrugator supercilii muscle, which is activated during expressions associated with anger (25).

\section{Discussion}

Research into leaked and ME is currently limited by several factors. Foremost, it is limited to manual visual examination or to computer vision methods which are computationally expensive, have restrictions of parameters, demand high image quality, specific camera perspectives and high frame rates. Second, different individuals employ different strategies to prevent leakage and inter-subject variability 


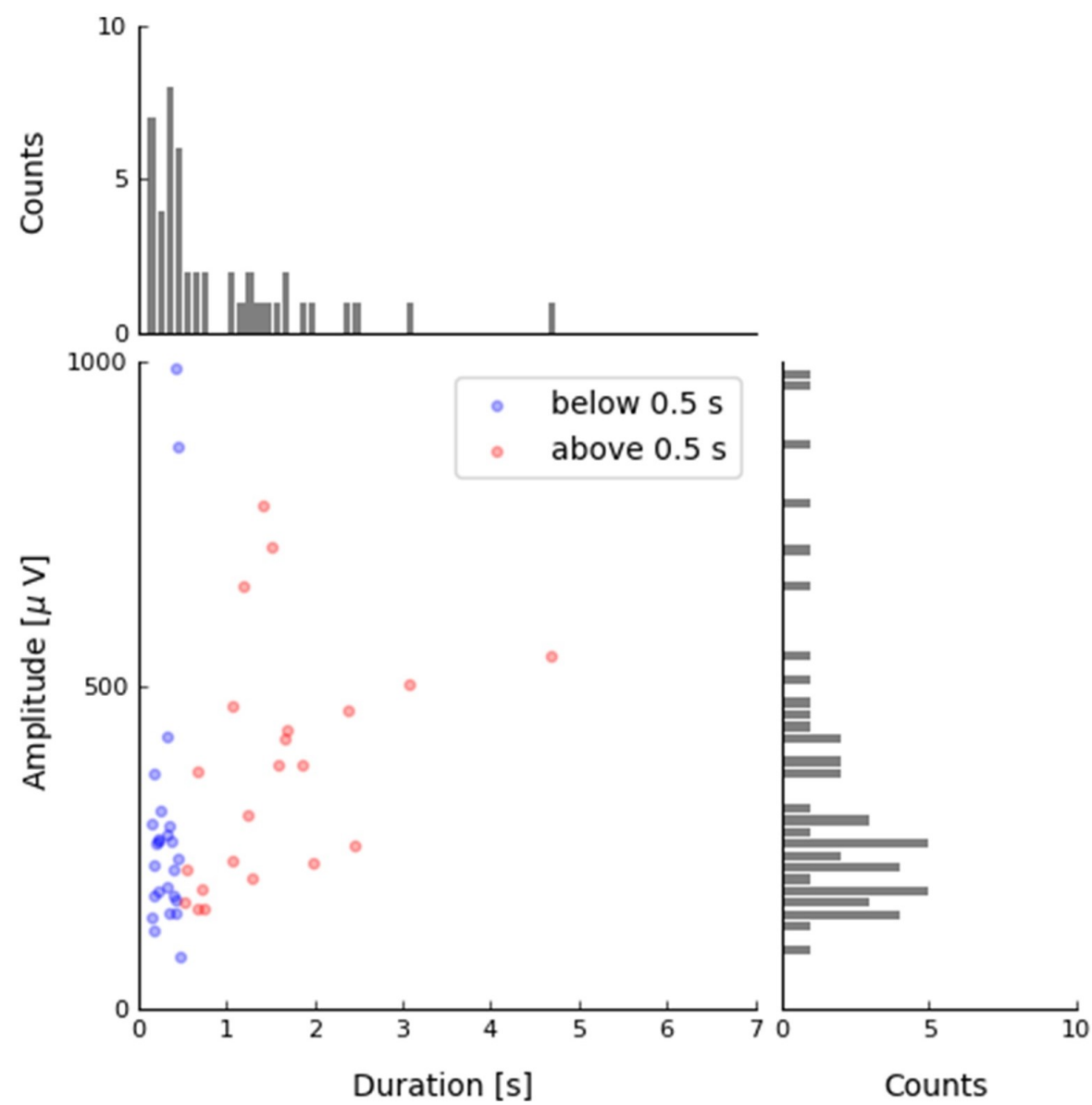

Figure 4. Leaked expressions duration and amplitudes. A histograms of (a) duration and (b) amplitudes. (c) cluster plot of amplitude versus duration. Expressions with total duration below and above $0.5 \mathrm{~s}$ are blue and red circles respectively.

is high. Third, experimental setups lack the original high-stake psychological scenarios needed to elicit a genuine leaked expression. The method we report here addresses some of these challenges. We discuss below these issues and their relevance to the current investigation. The low intensity and short time durations of leaked expressions are indeed a major challenge, and their detection is mostly achieved in a subjective manner using human coders. To facilitate systematic investigation of facial expressions, the Facial Action Coding System (FACS) was developed (15). FACS describes facial expressions and movements in Action Units (AU's) and Action Descriptors (AD's). AU's correspond to one or more muscles, defined as a muscle group, while the AD's correspond to several muscle groups. Each facial expression or head movement has an intensity score, from A being an almost non-noticeable trace, to E being the maximum intensity score. FACS trained human coding experts or machines are commonly employed to carry out classification. To date, classifications are based on subjective measures and it is common to use several coders to achieve a more robust classification. The FACS system is used in classification of facial expressions in general and specifically in ME (MEs). The Ekman group developed seven different ME related training tools: micro-expression training tool, subtle-expression training tool, ME profile, ME intensive, responding effectively home, responding effectively workplace and responding effectively law enforcement. These training tools are claimed to improve several skills: detection of concealed emotions, improvement of social intelligence, enhancement of relationships, developing the capacity of empathy and understanding others (ekm). To the best of our knowledge, reference (31) is the only report of micro-expression recording using sEMG. Extensive work was done 


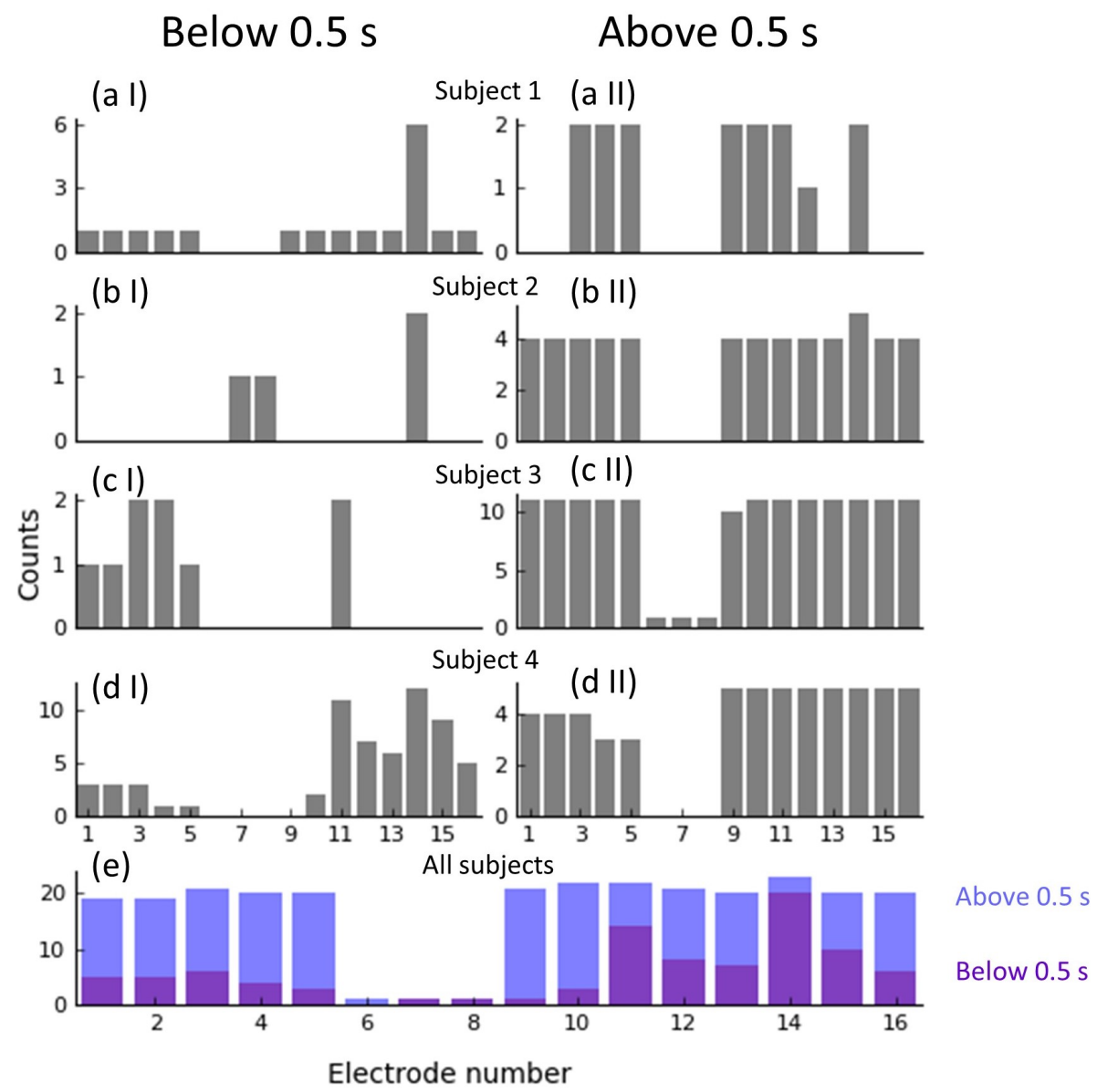

Figure 5. Histograms of activated electrodes. (a I - d I) Leaked expressions above $0.5 \mathrm{~s}$ and (a II $-\mathrm{d}$ II) Leaked expressions below $0.5 \mathrm{~s}$ for each subject individually. (e) Activation histogram for all subjects combined. Activation of electrodes above and below $0.5 \mathrm{~s}$ are in blue in purple, respectively.

in regard to facial expression recognition using sEMG. However, these studies did not targeting leaked expressions in general and ME specifically. Typically subjects were requested to mimic facial expressions $(9 ; 7 ; 25)$. As such, these expressions do not correspond with the intent of this study, the elicitation of leaked micro facial expressions. The difficulty in detecting ME using sEMG is related, most likely, to the low amplitude and relatively high noise caused by poor electrode-skin contact [13], and the fact that large electrodes restrict visibility for visual verification. The work presented in this paper provides the first successful recording of short leaked expressions using novel surface EMG electrodes. The electrodes are soft, thin and small $(3 ; 24 ; 21 ; 20)$, making them ideally suited for monitoring minute facial movements. We believe that the unique electrodes used in this study induced little (possibly even no) facial expressions attenuation, thus enabling to record, classify and characterize ME. Despite the many challenges presented in capturing leaked and micro expressions, they have huge potential in many fields and their physiological mapping is critically important. The USA Transportation Security Administration has employed a system for passengers' screening based largely on a ME named SPOT (34). Distributed across 161 airports in the USA with a total of 3000 trained agents, it is designed to identify suspects. Additionally, a trial program was issued in Heathrow airport based partially on SPOT, and the DHS is currently developing a system for detection of deception and hostile intent based on various non-invasive sensors (dec). All except the DHS deceit detection are based on human observers trained to detect ME. 
Deception detection is yet another important application. An objective and robust tool for the detection and classification of facial sEMG, combined with natural language processing and computer vision algorithms, can provide vital information regarding the internal state of the interrogated person. As a consequence, this increases the ability to steer the interrogation efficiently, and provide indication of concealment and repression of information, ultimately to achieve deception detection with unprecedented accuracy. The need for a robust and objective tool for deception detection spans from private security to police, military and intelligence agencies. Contemporary tools include the polygraph and Facial Action Coding System (FACS). Research regarding deceit detection by human observers show limited results. A meta-analysis performed by Bond and DePaulo indicate that the average accuracy for human deception detection lies just above chance (5). A study conducted by Shen et al. (32) assessed the ability of individuals to detect ME using two tools: The brief affect recognition test (BART) and the micro-expression training tool (METT), the results indicate there is an ability to detect basic facial-expressions (image) presented for a brief amount of time in a sterile experimental environment.

Wearable devices can be useful to some applications, while in others computer vision is indispensable. A skin wearable device will be useful for interrogators, while a remote, video-based system will be helpful in situations where the interrogated individual is not aware of the monitoring. Security cameras using computer vision and machine learning classifiers will have the potential to achieve unprecedented accuracy if proper classification is implemented. Investigative and intelligence units using the combined sEMG, video and voice analysis will have the ability to decrease the investigation time and increase data reliability. Leaked and ME may also be useful as biomarkers for neurological disorders. Macroexpressions were suggested as biomarkers for several neurological disorders such as Schizophrenia (28), Autism (29) and Parkinson's disease(33), and thus, it might be interesting to investigate the role of ME in this context.

\section{Conclusion}

Owing to their subtle nature, systematic study of ME was relatively limited and many of their properties remain unclear or under debate. Foremost is their precise definition, in particular, duration and location. Time duration of ME is a topic of on-going debate. Definitions in the literature vary from $40 \mathrm{~ms}$ to $0.5 \mathrm{~s}$ (36), raising the possibility that different types of ME exist. Uncertainty exists also in setting the lower boundary for ME duration. Wen-Jing Yan et al., suggested that the shortest events are in the $160 \mathrm{~ms}$ range, in contrast to Ekman's definition of $40 \mathrm{~ms}$ as a lower limit. In this investigation we demonstrated the ability to record highly localized and subtle leaked expressions using a new sEMG approach. The new method we present here may contribute in various domains ranging from generating reliable microexpression data-sets, enabling further research into the physiology of ME and possibly the development of better lie detectors.

Funding statement. This work was supported by a grant from the Israel Science Foundation (1355/17).

Competing interests. YH declares financial interest in X-trodes Ltd, which holds the licensing rights of the skin technology cited in this paper. The authors have no other relevant affiliations or financial involvement with any organization or entity with a financial interest in or financial conflict with the subject matter or materials discussed in the manuscript apart from those disclosed.

Ethical standards. All experiments on human skin were conducted on volunteers in accordance with relevant guidelines and regulations under approval from the Institutional Ethics Committee Review Board at Tel Aviv University. Informed consent was obtained from all subjects. Data

Author contributions. O.G. and Y.H. conceived and designed the experiments. O.G. performed the sEMG experiments, created the GUI for presenting the data and videos, the pipeline for extracting the facial expressions and analyzed the data. O.G. and Y.H. wrote the manuscript. Y.H. supervised the project. 


\section{References}

Deception Detection I Homeland Security.

Homepage - Paul Ekman Group.

Bareket, L., Inzelberg, L., Rand, D., David-Pur, M., Rabinovich, D., Brandes, B., and Hanein, Y. (2016). Temporary-tattoo for long-term high fidelity biopotential recordings. Scientific Reports, 6(April):25727.

Bengacemi, H., Abed-Meraim, K., Buttelli, O., Ouldali, A., and Mesloub, A. (2020). A new detection method for EMG activity monitoring. Medical \& Biological Engineering \& Computing, 58(2):319-334.

Bond, C. F. and DePaulo, B. M. (2006). Accuracy of Deception Judgments. Personality and Social Psychology Review, 10(3):214-234.

Borza, D., Danescu, R., Itu, R., and Darabant, A. (2017). High-speed video system for micro-expression detection and recognition. Sensors (Basel, Switzerland), 17(12).

Cai, Y., Guo, Y., Jiang, H., and Huang, M.-C. (2018). Machine-learning approaches for recognizing muscle activities involved in facial expressions captured by multi-channels surface electromyogram. Smart Health, 5-6:15 - 25. WEARABLE SENSOR SIGNAL PROCESSING FOR SMART HEALTH.

Canny, J. (1986). A Computational Approach to Edge Detection. IEEE Transactions on Pattern Analysis and Machine Intelligence, PAMI-8(6):679-698.

Cha, H., Choi, S., and Im, C. (2020). Real-time recognition of facial expressions using facial electromyograms recorded around the eyes for social virtual reality applications. IEEE Access, 8:62065-62075.

Charles Darwin, P. P. (1872). The Expression of the Emotions in Man and Animals. John Murray.

Cohn, J. F., Ambadar, Z., and Ekman, P. (2007). Observer-based measurement of facial expression with the Facial Action Coding System. The handbook of emotion elicitation and assessment, pages 203-221.

DesJardins, N. M. L. and Hodges, S. D. (2015). Reading Between the Lies. Social Psychological and Personality Science, 6(7):781-787.

Ekman, P. (2003). Emotions inside out : 130 years after Darwin's the expression of the emotions in man and animals. New York Academy of Sciences.

Ekman, P., Freisen, W. V., and Ancoli, S. (1980). Facial signs of emotional experience. Journal of Personality and Social Psychology, 39(6):1125-1134.

Ekman, P. and Freisen Wallace V. (1978). Manual for the Facial Action Code. CA: Consulting Psychologist Press.

Ekman, P. and Friesen, W. V. (1969). Nonverbal leakage and clues to deception. Psychiatry, 32(1):88-106.

Ekman, P. and Friesen, W. V. (1975). Unmasking the face: A guilde to recognizing emotions from facial expressions. Malor Books.

Haggard, E. a. and Isaacs, K. S. (1966). Micromomentary facial expressions as indicators of ego mechanisms in psychotherapy. Methods of research in psychotherapy, pages 154-165.

Hess, U. and Kleck, R. E. (1990). Differentiating emotion elicited and deliberate emotional facial expressions. European Journal of Social Psychology, 20(5):369-385.

Inzelberg, L., David-Pur, M., Gur, E., and Hanein, Y. (2020). Multi-channel electromyography-based mapping of spontaneous smiles. Journal of Neural Engineering, 17(2):026025.

Inzelberg, L., David Pur, M., Steinberg, S., Rand, D., Farah, M., and Hanein, Y. (2017). Wireless electronic-tattoo for long-term high fidelity facial muscle recordings. SPIE Proceedings 10194, Micro- and Nanotechnology Sensors, Systems, and Applications IX, 10194:101940U.

Inzelberg, L., Pur, M. D., Schlisske, S., Rödlmeier, T., Granoviter, O., Rand, D., Steinberg, S., Hernandez-Sosa, G., and Hanein, Y. (2018a). Printed facial skin electrodes as sensors of emotional affect. Flexible and Printed Electronics.

Inzelberg, L., Rand, D., Steinberg, S., David-Pur, M., and Hanein, Y. (2018b). A Wearable High-Resolution Facial Electromyography for Long Term Recordings in Freely Behaving Humans. Scientific Reports, 8(1).

Inzelberg, L., Rand, D., Steinberg, S., David-Pur, M., and Hanein, Y. (2018c). A Wearable High-Resolution Facial Electromyography for Long Term Recordings in Freely Behaving Humans. Scientific Reports, 8(1):2058.

Jiang, M., Rahmani, A., Westerlund, T., Liljeberg, P., and Tenhunen, H. (2015). Facial expression recognition with semg method. In 2015 IEEE International Conference on Computer and Information Technology; Ubiquitous Computing and Communications; Dependable, Autonomic and Secure Computing; Pervasive Intelligence and Computing, pages 981-988.

Li, X., Hong, X., Moilanen, A., Huang, X., Pfister, T., Zhao, G., and Pietikainen, M. (2018). Towards reading hidden emotions: A comparative study of spontaneous micro-expression spotting and recognition methods. IEEE Transactions on Affective Computing, 9(4):563-577.

Li, X., Pfister, T., Huang, X., Zhao, G., and Pietikainen, M. (2013). A Spontaneous Micro-expression Database: Inducement, collection and baseline. 2013 10th IEEE International Conference and Workshops on Automatic Face and Gesture Recognition, FG 2013.

Mattes, R., Schneider, F., Heimann, H., and Birbaumer, N. (1995). Reduced emotional response of schizophrenic patients in remission during social interaction. Schizophrenia Research, 17(3):249-255.

McIntosh, D. N., Reichmann-Decker, A., Winkielman, P., and Wilbarger, J. L. (2006). When the social mirror breaks: deficits in automatic, but not voluntary, mimicry of emotional facial expressions in autism. Developmental Science, 9(3):295-302. 
Merghani, W., Davison, A. K., and Yap, M. H. (2018). A review on facial micro-expressions analysis: Datasets, features and metrics. CoRR, abs/1805.02397.

Perusquia-Hernandez, M., Hirokawa, M., and Suzuki, K. (2017). A wearable device for fast and subtle spontaneous smile recognition. IEEE Transactions on Affective Computing, 14(8):1-1.

Shen, X.-b., Wu, Q., and Fu, X.-1. (2012). Effects of the duration of expressions on the recognition of microexpressions. Journal of Zhejiang University SCIENCE B, 13(3):221-230.

Smith, M. C., Smith, M. K., and Ellgring, H. (1996). Spontaneous and posed facial expression in Parkinson's Disease. Journal of the International Neuropsychological Society, 2(05):383-391.

Weinberger, S. (2010). Airport security: Intent to deceive? Nature, 465(7297):412-415.

Yan, W. J., Li, X., Wang, S. J., Zhao, G., Liu, Y. J., Chen, Y. H., and Fu, X. (2014). CASME II: An improved spontaneous micro-expression database and the baseline evaluation. PLOS ONE, 9(1):1-8.

Yan, W. J., Wu, Q., Liang, J., Chen, Y. H., and Fu, X. (2013a). How Fast are the Leaked Facial Expressions: The Duration of Micro-Expressions. Journal of Nonverbal Behavior, 37(4):217-230.

Yan, W. J., Wu, Q., Liu, Y. J., Wang, S. J., and Fu, X. (2013b). CASME database: A dataset of spontaneous micro-expressions collected from neutralized faces. 2013 10th IEEE International Conference and Workshops on Automatic Face and Gesture Recognition, FG 2013. 